\title{
Particles turbulence interactions in boundary layers
}

Plenary lecture presented at the 75th Annual GAMM Conference, Dresden/Germany, 22-26 March 2004

\author{
Alfredo Soldati * \\ Centro Interdipartimentale di Fluidodinamica e Idraulica and Dipartimento di Energetica e Macchine, Università di Udine, \\ Udine, 33100, Italy
}

Received 6 August 2004, revised and accepted 25 February 2005

Published online 4 July 2005

Key words particles, turbulence, coherent structures, boundary layer, turbophoresis, Direct Numerical Stimulation MSC (2000) 76F25, 76F65, 76T10, 76T15

\begin{abstract}
Turbulent dispersed flows in boundary layers are crucial in a number of industrial and environmental applications. In most applications, the key information is space distribution of particles which is known to be strongly non-homogeneous. Specifically, inertial particles distribute preferentially avoiding strong vortical regions and segregating into straining regions. The vortical boundary layer structures control momentum, mass, heat, and particle transfer. Coherent structures bring particles toward the wall and away from the wall and favour particle segregation in the viscous region giving rise to nonuniform particle distribution profiles which peak close to the wall. The reason for this behavior is particle inertia, which filters the high frequency turbulence fluctuations. The object of this work is to review the current understanding of turbulent boundary layer dynamics and to examine the mechanisms for particle transfer, segregation, and preferential distribution. The physical mechanisms discussed and proposed are based on Direct Numerical Simulations of turbulence and Lagrangian tracking of inertial particles.
\end{abstract}

(c) 2005 WILEY-VCH Verlag GmbH \& Co. KGaA, Weinheim

\section{Introduction and background}

Particle transport, dispersion, and segregation in turbulent flows are highly nonuniform and intermittent phenomena which are recognized to depend on the local dynamics of turbulence structures. A sound understanding and a thorough characterization of the mechanisms controlling particle transfer and segregation are of fundamental significance for a number of technological and environmental applications (e.g. mixing, combustion, depulveration, spray dynamics, pollutant dispersion, cloud dynamics...), and require deep comprehension of the interactions between particle dynamics and turbulent transport and mixing. In this context, I find the following statement by Godfrey Mungal [40] extremely clear and inspiring: Fluid motions [in turbulent boundary layers] are intermittent and have a strongly organized and coherent nature represented by the large scale motions. These motions, even though not exactly repeatable and only quasi-deterministic, control the transport of the dispersed species in such a way that the overall distribution will resemble not at all those given by methods in which these motions are ignored. In addition to these specific features of turbulent transport mechanisms, the complexity of dispersion phenomena increases when transported species are inertial particles which may not respond to small turbulence fluctuations as massless tracers. Since inertia is a low-pass filter, particles respond selectively to turbulence fluctuations so that the system fluid turbulence-inertial particles may give rise to peak phenomena such as long-term local particle accumulation or segregation. In the specific case of boundary layers, this leads to irreversible particle segregation at the wall [67]. In this paper, we will review the modelling framework of particle interaction with turbulence coherent structures in the specific context of the boundary layer. We will build up on our previous papers examining in detail particle transfer and segregation mechanisms and we will produce a unified viewpoint to explain macroscale particle segregation phenomena starting from local interactions of particles with turbulence structures.

Decades of extensive studies have clarified several issues concerning particle dynamics. It is well known how inertial particles are subject to the actions of the surrounding fluid and a number of papers have been produced which examine the relative values of the fluid forces acting on particles (see [37] for instance). However, if particle density is much larger than fluid density (as in many cases of interest: dispersed flyashes, droplets, and heavy sediments) the largest effects on particle motion are due to drag and inertia with only small quantitative corrections produced by all other fluid forces actions. Thus, if particle diameter is not negligibly small, inertia will influence strongly particle behavior. The trajectory of an inertial

\footnotetext{
* e-mail: soldati@uniud.it, Phone: +390432558020, Fax: +390432558027
} 


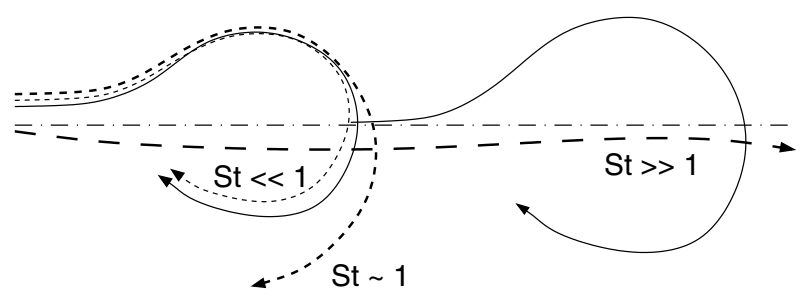

Fig. 1 Influence of particle relaxation time on particle trajectory. Small inertia particles follow precisely the flow; large inertia particles filter the space changes of velocity; intermediate inertia particles respond to the flow structures (see also [13]).

particle driven by the drag force in a vortical flow field is sketched in Fig. 1 in which solid lines represent tracer pathlines. In Fig. 1, the behavior of three different inertia particles is controlled by the particle time-scale - particle relaxation time which, from the steady-state balance between inertia and the linear Stokes drag, is defined as $\tau_{p}=\rho_{p} d_{p}^{2} / 18 \mu$, where $\rho_{p}$, $d_{p}$, and $\mu$ are particle density, particle diameter, and fluid dynamic viscosity, respectively. In Fig. 1, particles with different time-scale are subject to the same flow time-scale, $\tau_{f}$, defined on the basis of the inverse of vorticity. The ratio of the particle relaxation time to the flow time-scale defines the Stokes number as $\mathrm{St}=\tau_{p} / \tau_{f}$. Broadly speaking, particles act as a low-pass filter responding to the flow scales larger than their time-scale. If their time-scale is comparable to the fluid time-scale, particles may be propelled into specific flow regions and tend to sample the flow field in a preferential way.

This concept was demonstrated by Maxey [38] examining the behavior of swarms of inertial particles in a two-dimensional, periodic field of simple cellular vortices. In his numerical experiment, particles were settling under gravity and showed a tendency to sample the flow field preferentially. The same experiment was reproduced and results of particle preferential distribution are shown in Fig. 2: The effect is striking for this type of model steady flow and suggests that insights into real three-dimensional, time-dependent turbulent dispersions may be obtained by trying to identify the archetypal dynamics of the dominant flow structures.

Real three-dimensional time-dependent turbulent fields are characterized by vortical structures of largely different scales mutually interacting. Particles will be thus advected in a fashion which is not at all random and will assume a spatially intermittent distribution. This effect will be amplified or damped depending on the particle-to-flow time-scale ratio [7,8,16, 50,53,59].

Preferential segregation of particles is fundamental in a number of turbulent flow applications [7,53]. Yet, in the specific case of turbulent boundary layer, the local interaction between particles and turbulence structures leads to a remarkably unique macroscopic behavior, i.e. particle accumulation in the viscous sublayer [5,26,35,36,41,67]. This macroscopic behavior is due to the combined action of the many microscopic transfer phenomena which drive particles toward the wall and away from the wall. Since 1957, when Friedlander and Johnstone [17] in the context of deposition theory broadly differentiated between the behavior of large and small particles in the viscous sublayer, much attention was dedicated to investigation of particle wall transfer mechanisms. In 1975, Cleaver and Yates [12] proposed a sub-layer model based on the Reynolds analogy for particle transport in turbulent boundary layer for the deposition of small solid particles from a gas stream. According to this mechanism, particles are driven toward the wall and away from the wall by sweeps - coherent downwash of outer fluid to the wall - and ejections - coherent upwash of wall fluid toward the outer flow - which are instantaneous realizations of the Reynolds stresses - Q4 and Q2 type events respectively [66].

Of course, since early times efforts, Reynolds averaging of the Navier-Stokes equations assigned a crucial role to Q2 and Q4 events, yet it is only after the paper by Kline et al. [30] that they have been dignified as coherent structures together with the other time and space persistent flow phenomena in the boundary layer. The remarkable papers by Hussain [21,22] simplified the understanding of turbulence phenomena pinpointing that a clear identification and a correct definition of coherent structures were the key to understand possible archetypal dynamics in turbulent flows. This theoretical tool assisted by the rapidly increasing computational power, which finally made feasible long-desired, three-dimensional, timedependent, fully-resolved turbulent flow simulations, produced an entire branch of flourishing literature which gave new

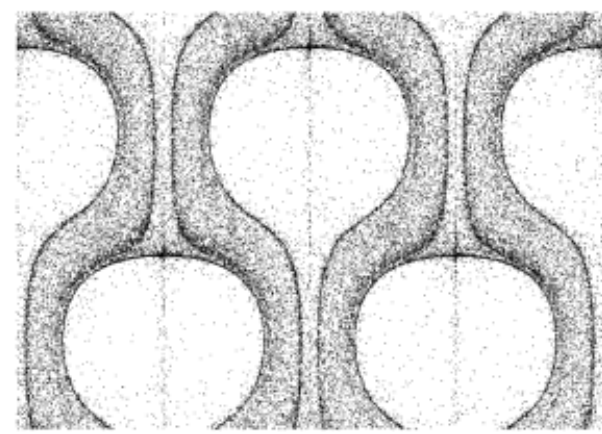

Fig. 2 Effect of inertia on particle preferential sampling of a periodic vortical twodimensional flow field reported by Maxey [38]: distribution of aerosol particles falling under gravity. The non-dimensional parameters characterizing aerosol motion are Stokes setting velocity for still fluid $W=\tau_{p} g=0.5$ and inertia parameter $\mathrm{St}=0.2$. 

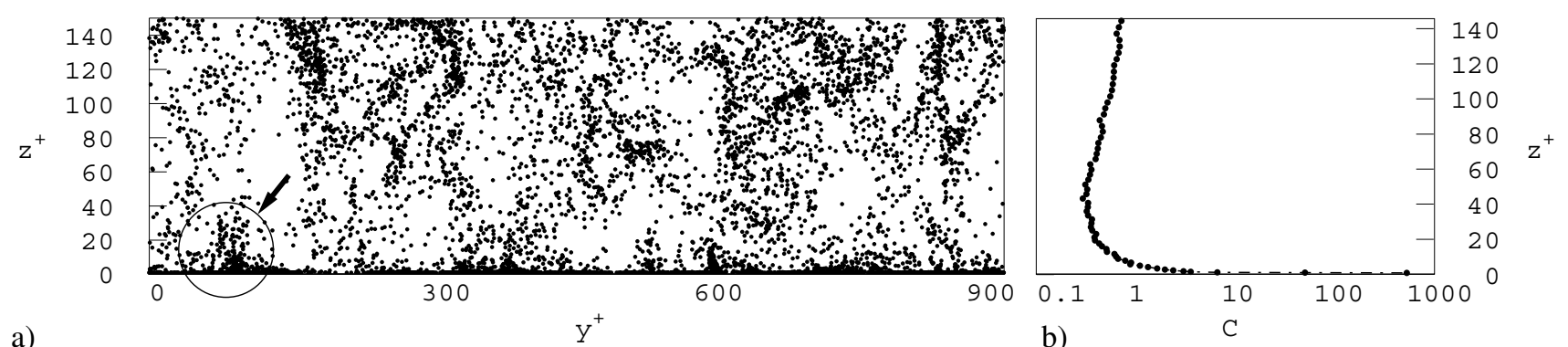

Fig. 3 Instantaneous distribution of particles characterized by $\tau_{p}^{+}=116.3$ at time $t^{+}=2700$. View of particle position in the $y z$-plane for $700<x^{+}<1000$ (a) and corresponding $x y$-plane average number density distribution as a function of the wall normal direction (b).

hopes in turbulence research by looking for coherent structures through the wealth of data available from Direct Numerical Simulation (DNS). In particular, many questions about the dynamics of turbulent boundary layers have been addressed and answered identifying the different characteristic coherent structures and proposing mechanisms to explain their generation process (the turbulence regeneration mechanism) [25,51,58,71].

There is a general consensus on the dynamics of turbulence structures in the boundary layer and the coherent structures have been precisely classified. The statistically most common coherent structures are single streamwise-oriented vortices, generally centred within the buffer layer. Quasi-streamwise vortices generate strongly coherent sweeps on the downwash side and strongly coherent ejections on the upwash side.

In the outer region, several recent investigations suggest that the most common vortex structures appear like hairpins whose legs are the counter-rotating quasi-streamwise vortices populating the near-wall region [51]. Neither these hairpins usually possess perfect spanwise symmetry nor the counter-rotating vortices have equal strength. Spanwise axisymmetric one-sided hairpins are also observed [18]. These new models revise and improve the classical concept of $\Omega$-shaped horseshoe vortices and are widely, though not totally, accepted. In recent papers, Zhou et al. [71] and Adrian et al. [1] proposed a new mechanism for turbulence regeneration cycle which is based on packets of hairpin vortices travelling at the same convection velocity, a new fundamental super-structure. Apparently, these super-structures populate all regions of the turbulent boundary layer and their characteristics fit well with most of previous quantitative observations from Kline et al. [30] up to the most recent. Furthermore, Adrian et al. [1] report that if we focus our attention only to the near wall region - i.e.less than 60 wall units from the wall - the phenomenology of the hairpin packet is very similar to the structures proposed by Schoppa and Hussain [56] and Jeong et al. [24].

Despite the great progress in the investigation of turbulence structures and dynamics, an equal effort was not produced in the area of turbulent dispersed flows. Several features of particle behavior in the boundary layer are broadly established, yet there are still many open issues concerning particle transfer mechanisms and particle segregation. In particular, even though the initial intuition by Cleaver and Yates [12] can be granted, quantitative evaluations are not yet broadly available. In addition, physical models which can explain why particles tend to accumulate at the wall [9,68], appear not completely explored. Still not fully understood are reasons and modalities under which, once at the wall, particles remain trapped in the low streamwise velocity regions at a distance from the wall not exceeding few wall units even when gravity does not play a role, both in horizontal flows with neutrally buoyant particles [26] and in vertical flows [9,65].

Several of the above mentioned phenomena are shown in Fig. 3(a) together with a number of features which can help us to focus on the process of particle dispersion and transfer in turbulent boundary layer. First, we observe that particles are not homogeneously distributed along the channel. In particular, particles tend to cluster around large vortical structures. From these clusters, particles are transported toward the wall, accumulating into specific "reservoirs" (one of these is indicated by the black circle) where concentration build-up occurs. These accumulation regions are characterized by flow streamwise velocity lower than the mean (see also [42,45] among others). Particles tend to stay long times in these low-speed regions so that eventually particle concentration increases near the wall. To quantify near-wall accumulation, the particle number density distribution is plotted as a function of the non dimensional distance from the wall $\left(z^{+}\right)$in Fig. 3(b). A logarithmic scale is used for particle number concentration to capture the detail of particle behavior in the proximity of the wall. The concentration profile is developing with time, and at the instant shot in Fig. 3(b), we observe that particle number density profile has developed a maximum well into the near-wall region $\left(0<z^{+}<20\right)$. This behavior can be viewed as the consequence of the turbulence non-homogeneity [50], and has been observed in a number of previous works [5,36,41].

The object of this work is to describe the complex causal relationship between the dynamics of turbulence structures near the wall, the local mechanisms driving particle transfer and the overall macroscopic observation on particle preferential distribution elaborating on our previous papers $[35,36]$. To this object, we will first review the details of the dynamics of 
turbulence regeneration cycle; then we will try to explain the phenomenology of particle transfer and preferential concentration in relation with the dynamics of turbulence coherent structures; and finally we will try to quantify from a statistical viewpoint particle preferential distribution in the viscous sublayer in connection with the flow topology.

\section{Numerical methodology}

The numerical experiments are performed using the pseudo-spectral solver of the fluid balance equations described in previous papers $[32,62]$ and Lagrangian particle tracking.

The flow into which particles are introduced is a turbulent Poiseuille channel flow of air, assumed incompressible and Newtonian. In the present study, we consider air with density $\rho=1.3 \mathrm{~kg} \mathrm{~m}^{-3}$ and kinematic viscosity $\nu=15.7 \times 10^{-6} \mathrm{~m}^{2} s^{-1}$. The reference geometry consists of two infinite flat parallel walls: the origin of the coordinate system is located at the center of the channel and the $x-, y-$ and $z-$ axes point in the streamwise, spanwise and wall-normal directions respectively. Periodic boundary conditions are imposed on the fluid velocity field both in streamwise and spanwise directions and no-slip boundary conditions are imposed at the walls. All variables are made dimensionless using the wall shear velocity $u_{\tau}$ and the fluid kinematic viscosity $\nu$ and are characterized by the superscript + . The shear velocity is defined as $u_{\tau}=\left(\tau_{w} / \rho\right)^{1 / 2}$, where $\tau_{w}$ is the mean shear stress at the wall. Calculations are performed on a computational domain of $1885 \times 942 \times 300$ wall units in $x, y$ and $z$ discretized with $128 \times 128 \times 129$ nodes. The shear Reynolds number is $\operatorname{Re}_{\tau}=u_{\tau} h / \nu=150$, where $h$ is the channel half height. Statistics of the flow field are consistent with previous simulations [28,34]. Some of the results related to the flow field will be presented for the same values of the parameters but from a simulation run on a coarser grid of $64 \times 64 \times 65$ nodes.

A Lagrangian particle tracking code coupled with the DNS code was developed to calculate particle paths in the flow field. The code interpolates fluid velocities at discrete grid nodes onto the particle position by means of $6^{\text {th }}$ order Lagrangian polynomials. Once the fluid velocity at particle position is known, the equations of particle motion are integrated in time by means of a $4^{\text {th }}$ order Runge-Kutta scheme. The interpolation scheme was compared both with spectral direct summation and with an hybrid scheme which exploits sixth order Lagrangian polynomials in the streamwise and spanwise directions and Chebychev summation in the wall-normal direction: Results showed good agreement between the three schemes. Particles are non-interacting, pointwise, rigid, spheres initially released at random locations within the computational box. We considered that a particle is elastically reflected away from the wall when its center is less than a distance equal to one particle radius from the wall. Particle density is in all cases large compared to fluid density. Particle momentum balance equation as discussed by Maxey and Riley [37] was simplified according to previous works [11,33] neglecting virtual mass, pressure gradient force, and Basset force. The only forces considered were inertia and drag which was computed according to the non-linear model as in [54].

We run different simulations presented by tracking four sets of $10^{5}$ particles with $\mathrm{St}=\tau_{p}^{+}=0.2,1,5$, and 25 . In these work we will examine the influence of turbulence structures on particle transfer in the dilute flow approximation. We are currently examining complementary databases in which we consider particles feedback on the flow field. Since our main aim is to identify a theoretical frame-model for particle transfer and dispersion in turbulent boundary layer, we rely on the most simplified simulation setting. We believe that in the case of dilute dispersion of particles with density much larger than the fluid, all other effects including two-way coupling will be only a quantitative correction to the main phenomena investigated. Other previous findings support these assumptions [26,43].

\section{Turbulence structures in the boundary layer and particle dynamics}

\subsection{Turbulence wall structures}

In a turbulent boundary layer, momentum, heat, and mass transfer are controlled by the instantaneous realizations of the Reynolds stresses. Ejections and sweeps - Q4 and Q2 type events, respectively - control momentum transfer at the wall and are also well correlated to heat transfer [27,44] and mass transfer [14] at the wall. Specifically, ejections bring the low-momentum fluid close to the wall into the outer region whereas sweeps bring the high-momentum fluid from the outer flow into the wall region. A complete characterization of sweeps and ejections and of their generation mechanisms is thus fundamental to understand the physics of turbulence structure at the wall and to explain the effect produced on particle dynamics. The snapshot shown in Fig. 4 visualizes sweeps, ejections, and their action of momentum transfer to the wall. In this figure, flow is from left to right along the $x$ direction. Sweeps (gold lumps) and ejections (blue lumps) are identified with the same value of the instantaneous stress isosurface at $u^{\prime} w^{\prime}=-3$ in wall units. To visualize the correlations "sweep-high shear stress" and "ejection-low shear stress" the wall is colored with the intensity of the instantaneous shear stress at the wall - blue is low and red is high. It is apparent that low shear-stress regions correspond to the ejections, whereas high shear-stress regions correspond to the sweeps. 


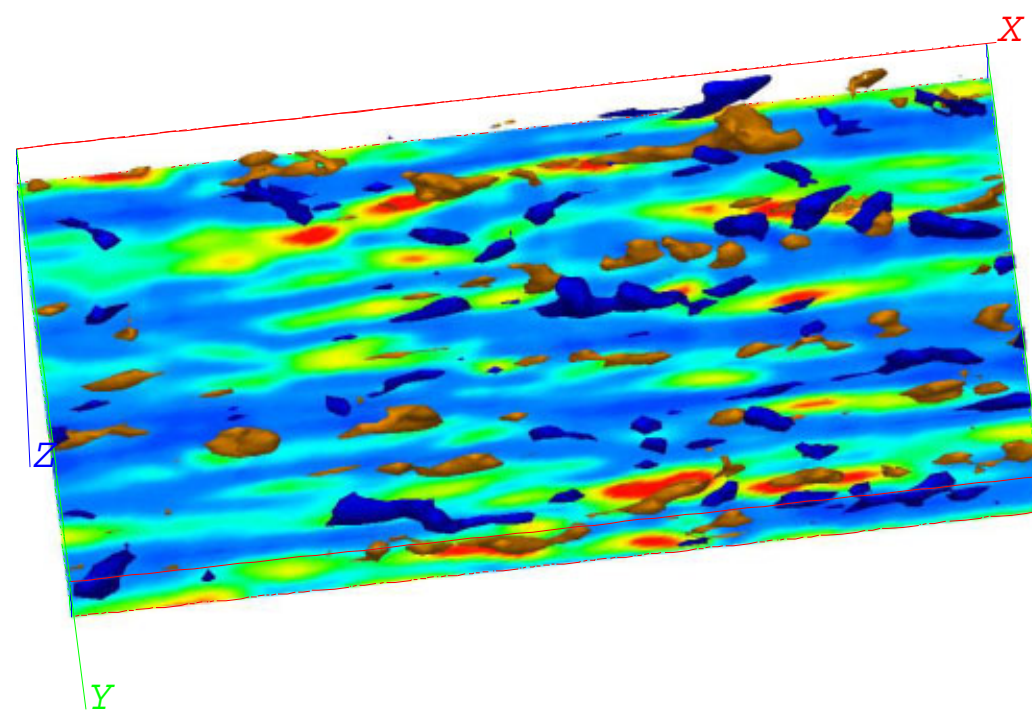

Fig. 4 Snapshot footprint of the wall shear-stress with corresponding sweep and ejection events in the whole computational domain. At the wall, red indicates high shear-stress; blue indicates low shear-stress. Gold 3D regions are isosurfaces characterizing sweeps whereas blue $3 \mathrm{D}$ regions characterize ejections. Isosurfaces are traced at $u^{\prime} w^{\prime}=-3$ in dimensionless units.

Sweeps and ejections are just a chain ring of wall turbulence regeneration cycle, and there is still some uncertainty about the mechanisms which generate and maintain the sweep/ejection events. They appear to be generated by the quasistreamwise vortices which populate the near wall region. Quasi-streamwise vortices have been the object of a number of works starting with Kline et al. [30], and there is a general consensus about their characteristic length (about 200 wall units) and spacing (about 400 wall units) $[24,25,30,56]$. Quasi-streamwise vortices are slightly tilted away from the wall and are responsible for pumping fluid towards and away from the wall. Clockwise and counterclockwise rotating vortices are slightly tilted upward - about $9^{\circ}$ average [56]; and are also slighthy tilted about $4^{\circ}$ left and right, respectively. The streamwise vortices may be identified by using pressure, vorticity, or other indicators $[4,15,20]$. A broadly-used method for identification exploits the streamline rotation vector $\Omega$ to visualize vortices as flow regions where the rate-of-deformation tensor $\partial u_{i} / \partial x_{j}$ exhibits complex eigenvalues $[10,23,46]$. From a physical viewpoint, the vector $\Omega$ represents strength and direction of the rotation of the streamlines. For further details, see Appendix 4.

In Fig. 5, two counter-rotating vortices, identified by one isosurface of $\Omega$, are shown together with the ejections and sweeps they generate. The elongated red and pale blue structures are two isosurfaces with the same absolute value of $\boldsymbol{\Omega}$ (and opposite sign) and indicate clockwise rotating (red) and counterclockwise rotating (pale blue) vortices. Flow is going from bottom left to top right and vortices appear tilted away from the wall by the mean strain rate. The blue lumps of fluid in between the two vortices are ejections and the green lumps of fluid outside the two vortices are sweeps. Ejections and sweeps also appear stretched streamwise and affected by the mean strain rate. Owing to the continuous action of the quasi-streamwise vortices in generating sweeps and ejections, regions between two vortices such as those shown in Fig. 5 are characterized by a streamwise velocity lower than the mean low-speed streaks, whereas the regions outside the two vortices are characterized by a streamwise velocity higher than the mean high-speed regions.

Many quasi-streamwise vortices are usually associated with one single low-speed streak. Low-speed streaks are sinuous regions about 1000 wall units long and are more coherent than high-speed regions.

In Fig. 6, a 450 wall units long piece of one low-speed streak is shown, flanked by two counter-rotating quasi-streamwise vortices. The red isosurface identifies a streamwise velocity value of $0.56 U_{c}$, where $U_{c}$ is centerline velocity, which is broadly considered the advection velocity of the low-speed streak [29]. In this figure, the action of the quasi-streamwise vortices in lifting up the low-speed streak is clear. Streamwise vortical structures overlap streamwise as a staggered array, as was clearly demonstrated by Schoppa and Hussain [56-58]. One single low-speed streak has a longer life than quasi-streamwise vortices and survives a number of vortex generations. It has been shown [29] that the generation of the quasi-streamwise vortices is associated with lateral instabilities producing changes in the shape of the low-speed streak surface. In recent papers, Schoppa and Hussain [56-58] suggested that wall turbulence is dominated by a cycle in which low-speed streaks generate quasi-streamwise vortices, which in turn generate ejections and sweeps. These finally contribute to maintain the low-speed streaks.

Thus, the view of evolutionary dynamics of boundary layers structures changes perspective in that streaks are considered responsible for the initial generation of quasi-streamwise vortices. Based on this view, Schoppa and Hussain [56] suggest different strategies for turbulence control. The most interesting strategy seems to be stabilization of the low-speed streaks by means of large-scale forcing motions. In practice, a low-speed streak which is more stable to spanwise perturbations would 


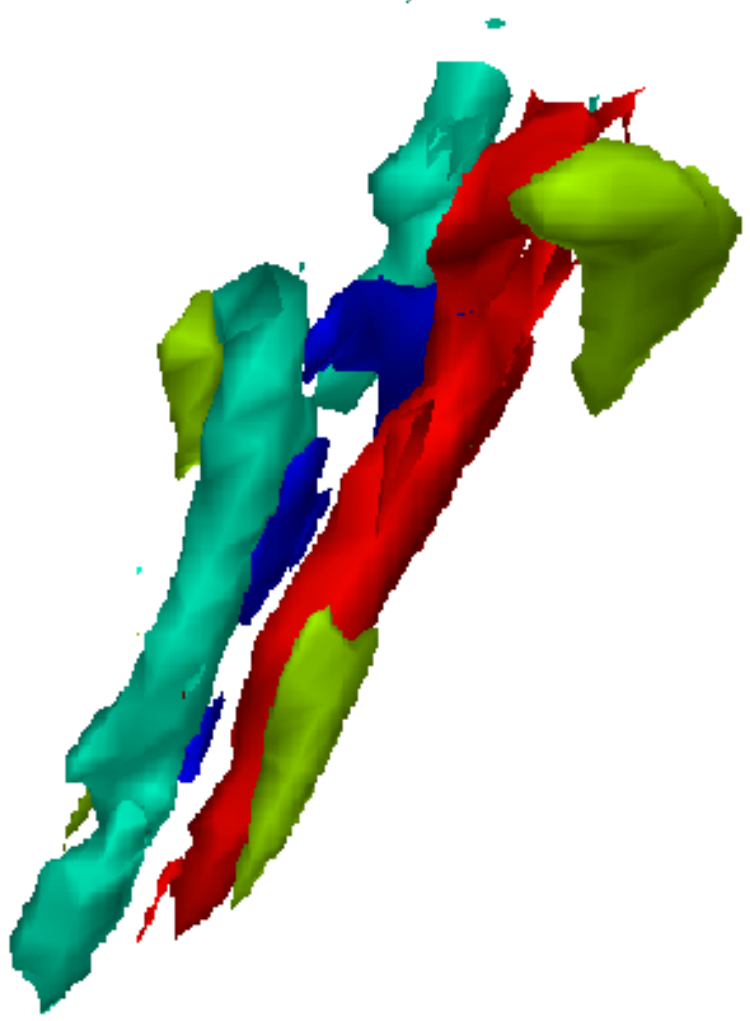

Fig. 5 Quasi-streamwise counter-rotating vortices together with ejections and sweeps. Quasi-streamwise vortices extend for about $200-300$ wall units. Two isosurfaces of the same absolute value of $\boldsymbol{\Omega}$ indicate clockwise rotating (red) and counterclockwise rotating (pale blue) vortices. Sweeps and ejections are indicated by green and blue, respectively.

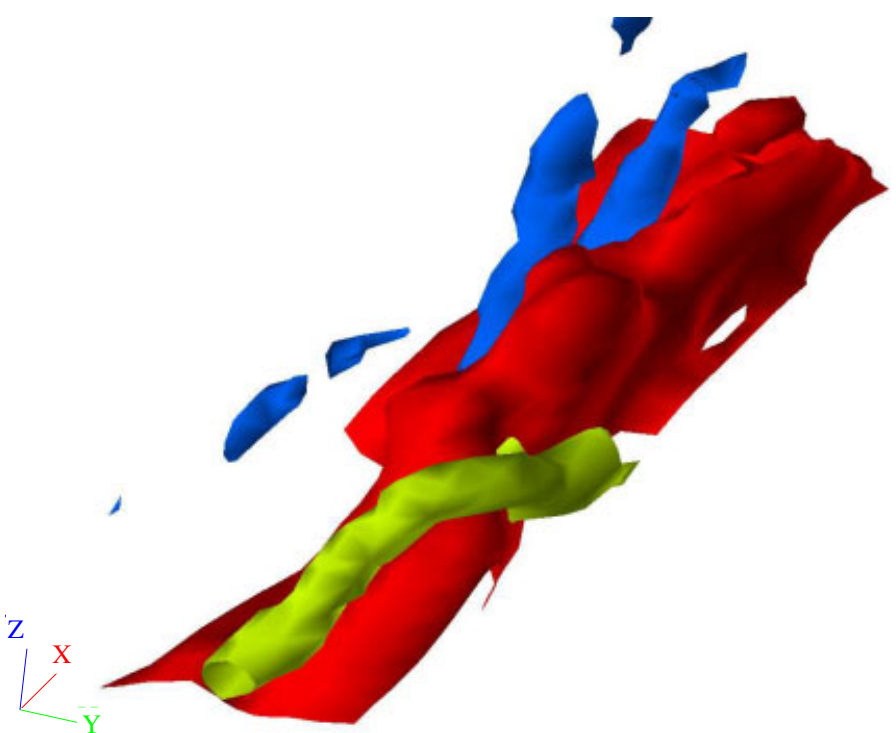

Fig. 6 Two counterrotating quasi-streamwise vortices onto a single low-speed streak (red). Green isosurface of $\boldsymbol{\Omega}$ indicates clockwise rotating vortex, blue isosurface of $\Omega$ indicates counterclockwise rotating vortex. Picture covers a streamwise window about 450 wall units long.

reduce its meandering and reduce the tripping frequency of quasi-streamwise vortices eventually reducing the frequency and the intensity of turbulence production events - i.e. sweeps and ejections.

\subsection{Particle dynamics}

There is experimental and numerical evidence that heavy particles in turbulent boundary layer have a tendency to migrate toward the wall under the turbophoretic drift $[5,8,39,50,67]$. Fig. 7 shows the particle number density concentration plotted as a function of the non dimensional wall distance $z^{+}$. A logarithmic scale is used to capture the detail of particle distribution in the near-wall region. Particle number concentration is normalized to the initially uniform concentration and is calculated after 1125 time wall units. As discussed by Portela et al. [49], particle distribution is not yet statistically steady. Regardless of particle size, number concentration is non-uniform along the wall normal coordinate, the trend being most pronounced for larger particles. In particular, the concentration profile appears to reach a maximum very close to the wall. This behavior can be viewed as the consequence of non-uniform turbulence advection mechanisms, the intensity of which decreases to very low values in the near wall region.

Number concentration is non-uniform also in the wall parallel direction, with particles segregated preferentially in regions characterized by streamwise velocity lower than the mean $[16,42,43,45]$. Fig. 8 shows the instantaneous distribution of $\tau_{p}^{+}=25$ particles in the region between the wall and $z^{+}=3$. The tendency of inertial particles to accumulate in the low-speed regions may support a possible use of particles as smart roughness [43]. In real situations, characterized by flow field modulation by the particles, the presence of particles would increase the inertia of the low-speed streaks. Since lowspeed streak stability to lateral perturbation has an impact on the wall turbulence regenerat cycle $[24,55,58]$, the presence of specific inertia or size particles in turbulent boundary layer might be exploited to tune wall transfer mechanisms. 


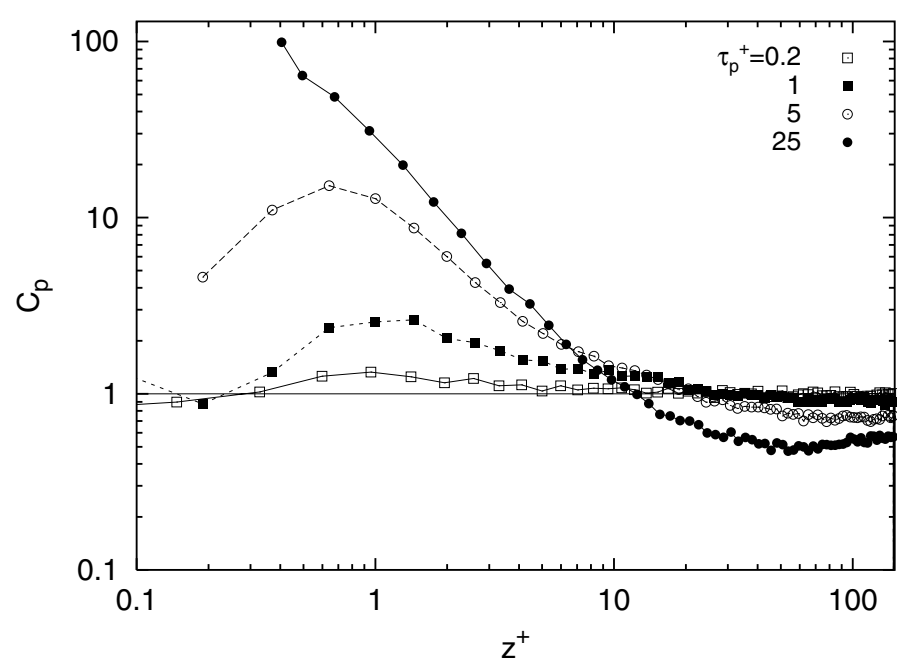

Fig. 7 Macroscopic effect of particle interaction with turbulence structures. Particle number density distribution calculated from one way coupling simulations for particle time scales equal to $0.2,1,5$ and 25 against non dimensional distance $z^{+}$from the wall (logarithmic scale). The concentration profile reach a maximum very close to the wall; the non uniform trend is most pronounced for larger particles.

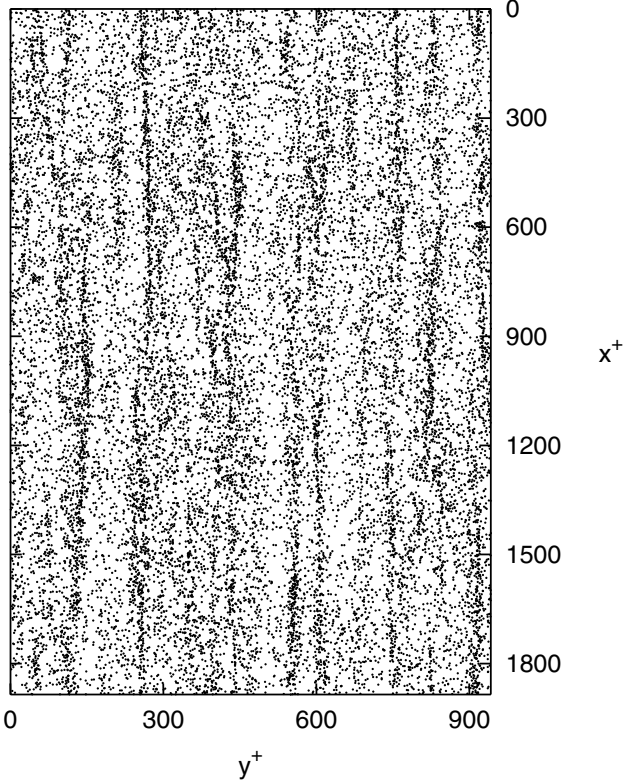

Fig. 8 Top view of particle distribution in the boundary layer. Note accumulation of particles in specific regions which correspond to the low-speed streaks.

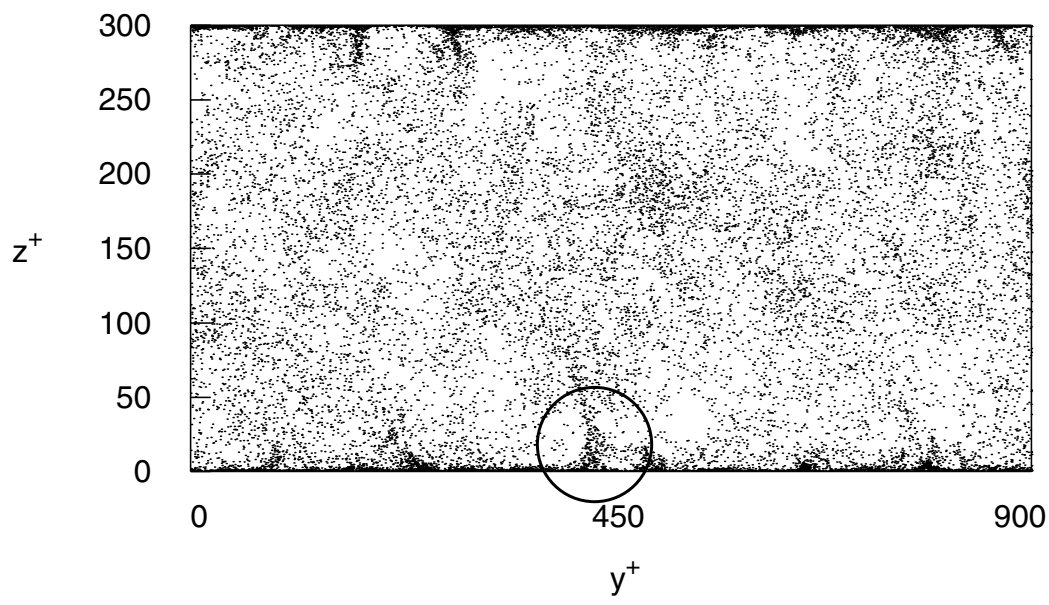

Fig. 9 Cross section of instantaneous particle distribution in the $y-z$ plane for $\Delta x^{+}=400$.

In Fig. 9 we show an instantaneous cross section in the $(y, z)$ plane of particle distribution. Specific regions of particle accumulation are clearly visible. These regions identify the main gateways for particle transfer to the wall region. In a previous work [35], we examined the relationship between particle fluxes in and out the wall layer and momentum fluxes at the wall. We found that particles are transferred almost exclusively by strongly coherent sweeps and ejections: Specifically, a strong correlation exists between sweep events and particle flux toward the wall, and between ejection events and particle flux toward the outer flow. This correlation is almost perfect for smaller particles and somehow weaker for larger particles. In other words, if a small particle travels toward the wall, it is entrained in a sweep whereas if the particle travels away from the wall, it is driven by an ejection. For larger particles, most of the particles are still transferred by sweeps and ejections but a higher proportion of particles with positive wall normal velocity appears in fluid environments characterized by negative wall-normal velocity, and viceversa. In particular, for the larger sets of particles, the fraction of particles travelling toward the wall in a non-sweep environment is smaller than the fraction of particles travelling away from the wall in a non-ejection environment [35]. 
This behavior is easily attributed to the local particle Stokes number which increases along the Lagrangian trajectory of the particle which, while travelling toward the wall, interacts with smaller and smaller flow structures [48]. The characteristic time-scale of turbulent structures scales linearly with wall distance and decreases progressively as the structures lie closer to the wall. The strongly coherent sweeps which transfer effectively particles to the wall are generated by the forward-end of the mature quasi-streamwise vortical structures which is located in the buffer layer. Larger particles have a larger time-scale and filter out the effects of the smaller fluid scales. Thus, the larger momentum gained by the large particles in the strongly coherent sweep is able to drive them to the wall and may be sufficient to let the particle bounce elastically off the wall, crossing the smaller scale structures in the vicinity of the wall unable to further modify the trajectory of the particle.

Particle Stokes number is also responsible for particle accumulation under the low-speed streaks. Fluid obeys continuity and fluid fluxes to the wall must be balanced by counterfluxes away from the wall. Particles, however, behave somehow as a compressible fluid and may accumulate as precisely according to what is happening to the small swarm of particles circled in Fig. 9, which is undergoing a strongly coherent phenomenon. Fig. 10(a) shows a cross section of the flow field in the region of particle accumulation. Vectors represent velocity components in the plane and color isocontours show the values of the streamwise velocity component. A strongly coherent ejection is present in the middle of the figure and indicates the position of the low-speed streak which appears lifted and flanked by two counter-rotating vortical structures. We identified particle position with the circles - larger than the real scale for visualization purposes. Blue particles have wall normal velocity directed away from the wall $\left(w_{p}>0\right)$ whereas purple particles have wall-normal velocity directed toward the wall $\left(w_{p}<0\right)$. In the outer region, say above $z^{+}>100$, there is no evident correlation between particle wall-normal velocity and fluid streamwise velocity. Approaching the wall, however, virtually all particles entrained in the ejection - streamwise velocity lower than the mean - have positive wall normal velocity indicating an extremely focused and coherent event. Particles approaching the wall are entrained in the two sweep events - yellow regions. Depending on the momentum they acquire, particles directed towards the wall may reach it or may follow the flow streaklines approaching the ejection to be re-entrained in the outer flow.

In Fig. 10(b) we show the same cross section of the flow field but this time we focus on the flow structures. We use streamwise vorticity isosurfaces to identify the pair of quasi-streamwise vortices responsible for the flow field examined in Fig. 9. The green isosurface identifies the counter-clockwise rotating quasi-streamwise vortex, whereas the pale blue isosurface identifies the clockwise rotating quasi-streamwise vortex. Particle color code is the same as in Fig. 10(a). Few particles are present in the vortex regions since they are swept in the region between the vortices which is, again, a low-speed streak.
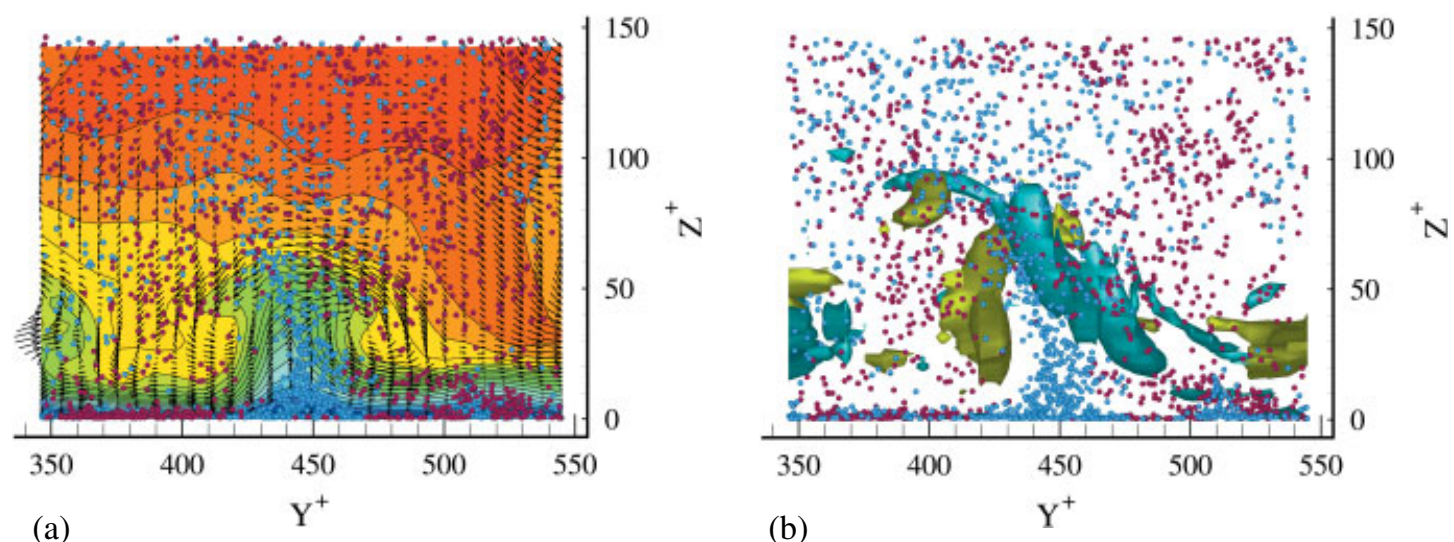

Fig. 10 Cross section of the flow field and front view of particles in the region of particle accumulation (a) and front view of particles and structures in the region of particle accumulation (b).

\subsection{Particle trapping and re-entrainment mechanisms}

With reference to Figs. 10(a),(b), we can tell the following story for particles initially in the outer flow: If a particle is captured by a sweep, it moves along a curved trajectory around the quasi-streamwise vortex generating the sweep, approaches the wall and moves between the vortex and the wall. During this phase, the particle may touch the wall or not, depending on its inertia. Then, the particle is on the upwash side of the vortex and is subject to the influence of the ejection. The next step involves trespassing the lifted low-speed streak and exiting from the wall layer. Particles should thus migrate toward the surface of the lifted low-speed streak, which is an ejection-like environment, and find an ejection strong enough to drive them into the outer flow. However, the story of particle accumulation at the wall has a further complicative effect which is 


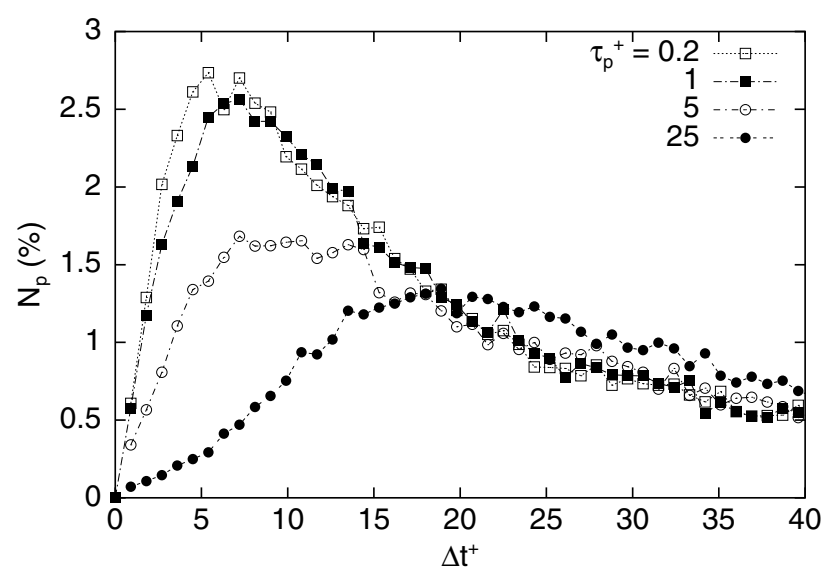

Fig. 11 PDF of the particle residence time in the viscous sublayer $\left(z^{+}<5\right)$.

linked to the time-scale of the turbulence regeneration cycle. We produced a model for particle trapping at the wall based on the joint examination of particle dynamics together with the dynamics of the turbulence structures populating the near wall region of the boundary layer. According to this model [35], it is crucial the presence of a secondary wall structure which was described by Brooke and Hanratty [6] and by Bernard et al. [2]. This structure is the rear-end of a quasi-streamwise vortex which prevents particles in proximity of the wall to be re-entrained by the pumping action of the large, farther from the wall, forward-end of an upstream, following quasi-streamwise vortex. The local flow structure produced by this couple prevents a number of particles which entered the wall layer from being entrained toward the outer flow. In particular, even though the strongly coherent sweep events required to drive particles to the wall are associated with strongly coherent ejections capable of driving particles toward the outer flow, the simultaneous presence of the offspring (or secondary) vortex acts as to reduce the width of the ejection channel. In practice, only particles which enter the wall-layer with specific trajectory curvature may be re-entrained into the outer flow.

According to this scenario, particles are either re-entrained immediately by the same vortex which brings them to the wall or they are confined for very long times in the viscous region [41]. We observed that most of the particles driven to the wall by a sweep are not re-entrained to the outer flow: They are bound to remain in the viscous wall layer for long times and slowly diffuse to the wall [41]. Particles can be re-entrained only if their behavior is consistent with the motion and the effects of coherent wall structures, which assist in resuspension, by conveying particles from the wall region to the outer flow through fluid ejections. We will now examine preliminary results on the influence of inertia on the mechanisms of particle re-entrainment from the viscous sublayer.

Quasi-streamwise vortices control particle re-entrainment via the ejections they generate. However, larger particles will require larger momentum than smaller particles. We thus tried to link the inertia of the re-entrained particle to the size of the structure responsible for re-entrainment. Specifically, for each particle entering the viscous sublayer - threshold fixed at $z^{+}=5$ - we started the time counter. We stopped the time counter when the particle exited the viscous sublayer. In Fig. 11, we show the probability density function that a particle of the time scale examined $\left(\tau_{p}^{+}=0.2,1,5,25\right)$ spend a specific residence time $\Delta t^{+}$in the viscous sublayer. All curves are similar for times larger than 20. Each curve has a peak: about 5 for $\tau_{p}^{+}=0.2$; about 7 for $\tau_{p}^{+}=1$; between 7 and 13 for $\tau_{p}^{+}=5$; and about about 18 for $\tau_{p}^{+}=25$.

It was shown [41] that, if a particle is trapped in the viscous sublayer, its residence time can be from hundred to thousand time wall units. Thus, a short residence time may indicate that a particle penetrating the viscous sublayer gains the exit just riding the same vortical structure which brought it inside in the first place. Considering that the characteristic time-scale of turbulent structures scales linearly with wall distance and decreases progressively as the structures lie closer to the wall, examining Fig. 11 we first observe that large particles may exit the viscous sublayer quickly only if re-entrained by large structures. Second, re-entrainment mechanisms for small particles are dominated by those structures with turnover time of about 5 time wall units. Apparently, a large particle is not able, once it is close to the wall to correct its trajectory sharply enough to catch the next ejection. Large particles may participate to the same sweep/ejection chain event only if it is generated by a large enough quasi-streamwise vortex.

\subsection{Flow topology and particle distribution}

In this section we will try to provide a clear methodology to quantify particle preferential distribution in the viscous sublayer. Specifically, we aim at investigating on the relationship between particle distribution and flow topology as discussed by Rouson and Eaton [52], to demonstrate the role of specific critical flow regions at the wall in attracting and segregating particles [48]. 
As already observed $[26,43,70]$ particle position in the wall region correlates well with low-speed streaks, which are characterized by negative values of the streamwise velocity fluctuation, $u^{\prime}$. Even though low-speed streaks are ejection-like environments, most of the particles do not leave the wall region but rather they remain trapped for long time. We established that a particle can exit the wall region if and only if it is entrained by a coherent vortical structure. We will try to use global identifiers to correlate particle distribution in the viscous sublayer with coherent structures on a statistical base. We used this methodology in a number of different flow types, ranging from boundary latyers [35] to jets [7,53].

In particular, we will relate particle non-homogeneous accumulation to coherent flow structures, according to the general classification scheme proposed by [10] and [3]. This classification scheme groups all elementary three-dimensional flow patterns basing on the three invariants of the velocity gradient tensor $u_{i, j}$ :

$$
\begin{aligned}
P & =u_{i, i}, \\
Q & =\frac{1}{2}\left[\left(u_{i, i}\right)^{2}-u_{i, j} u_{j, i}\right]=-\frac{1}{2} u_{i, j} u_{j, i}=\frac{1}{2}\left(\Omega_{i j} \Omega_{i j}-S_{i j} S_{i j}\right), \\
R & =-\lambda_{1} \lambda_{2} \lambda_{3},
\end{aligned}
$$

where the RHS of Eq.(2) holds for incompressible flow fields in which we further observe that $P=0$. Here $\Omega_{i j}=$ $\frac{1}{2}\left(u_{i, j}-u_{j, i}\right)$ and $S_{i j}=\frac{1}{2}\left(u_{i, j}+u_{j, i}\right)$ are the antisymmetric and symmetric components of the velocity gradient whereas $\lambda_{1}, \lambda_{2}$, and $\lambda_{3}$ are the eigenvalues of $u_{i, j}$. The velocity gradient tensor has been widely used to describe typical local deformations of fluid elements. Its invariants determine, for instance, if the deformations produce vorticity stretching along a specific direction (possibly yielding tubular vortices) or if they produce structures that are flattened out in one direction while expanding in the other two (possibly yielding pancake-like structures). In an effort to give a physical insight, we can consider as an example the quantity $Q$. Limited to incompressible flows, $Q$ represents the local balance between vorticity (related to $\Omega_{i j}$ ) and strain rate (related to $S_{i j}$ ). Thus, a fluid point characterized by positive values of $Q$ indicates the presence of high vorticity, whereas for negative values of $Q$ the local flow is dominated by straining motions [23]. All the incompressible flow topologies which may be classified using the $(Q, R)$-plane [3] are shown in Fig. 15 in Appendix. Four regions can be identified: two vortical flow regions, the so-called stable focus/stretching $(I)$ and unstable focus/compressing critical modes $(I I)$, and two convergence regions, the so-called stable node/saddle/saddle $(I I I)$ and unstable node/saddle/saddle critical nodes $(I V)$. Further critical points can be identified along the $Q$-axis and the $\Delta=0$ line: the so-called center/no flow $(\alpha)$, stable star node/saddle/saddle $(\beta)$, stable line node-saddle/unstable line node-saddle/no flow $(\gamma)$ and unstable star node/saddle/saddle $(\delta)$. In this work, we will exploit this classification system to elucidate the relationship between particle distribution and near-wall vortices.

Following Rouson and Eaton [52], we computed $Q$ and $R$ for the fluid at each grid point in the viscous sublayer. Then, we conditionally sampled $Q$ and $R$ at particle position to determine particles preference for or against any of the aforementioned topologies.

Fig. 12 shows the resulting joint probability density function (PDF) of $Q$ and $R$ in the viscous sublayer. PDFs were calculated over 400 instants of the flow field to consider only those events with significant statistical occurrence. We discuss results only for the $\tau_{p}^{+}=1,5$, and 25 particles. The set of smaller particles $\left(\tau_{p}^{+}=0.2\right)$ did not show significant preferential distribution compared to the flow, as already observed in Fig. 7. The most probable value of $Q$ and $R$ is zero for all the instants. The PDF sampled for the fluid at grid points (Fig. 12(a)) shows that the preferred quadrants correspond to the stable focus/stretching $(I I)$ and the unstable node/saddle/saddle $(I V)$ topologies. Also, the lines of constant PDF asymptote toward the $\delta$-curve, which represents the tail of the tear-drop shaped $(Q, R)$-distribution. The PDF sampled at $\tau_{p}^{+}=25$ particle positions (Fig. 12(b)) occupies a relatively small area, centered around the origin of the $(Q, R)$-plane, indicating that larger particles tend to avoid the strongest vortical regions (corresponding to quadrants $I$ and $I I$ in Fig. 15) as well as the convergence regions corresponding to quadrant $I V$ in Fig. 15. Only convergence regions (corresponding to $I I I$ quadrant in Fig. 15) seem to be little affected by the preferential sampling of these particles. The behavior of the $\tau_{p}^{+}=5$ particles (Fig. 12(c)) is characterized by a broader PDF which would suggest a behavior similar to that of fluid particles in the proximity of the wall. The PDF sampled at $\tau_{p}^{+}=1$ particle positions (Fig. 12(d)) indicates a behavior similar to that of the fluid. Results obtained by Rouson and Eaton [52] for the PDF sampled at particle position are qualitatively similar despite some quantitative differences possibly due to the several differences in the simulations parameters (particularly we refer to the inertia of the particles examined). Rouson and Eaton [52] considered rather large particles, being interested in analyzing their behavior in the entire channel. We focused our analysis on small- to moderate-inertia particles, in order to match the ever-decreasing flow time scales which particles encounter when approaching the wall [48].

The topological analysis seems to suggest that, very near the wall, strongly coherent vortical structures are depleted of particles as would be if the local flow is producing particle non-homogeneous concentration [52]. This result is relevant and yet it gives little specific information about the wall regions where particle build-up occurs. To identify these regions we need a different identification criterion [48]. 


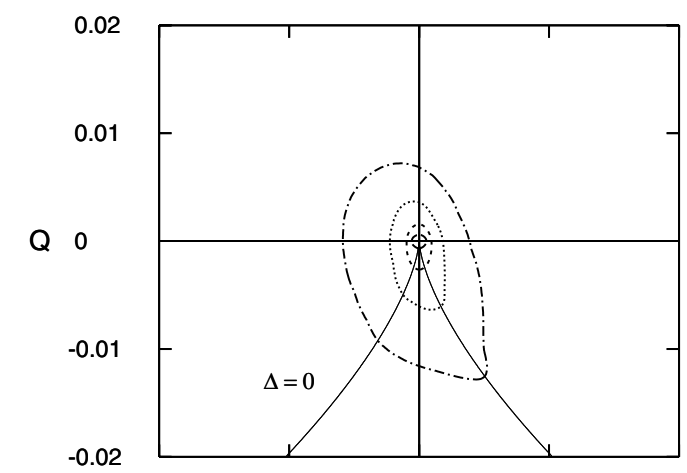

(a)

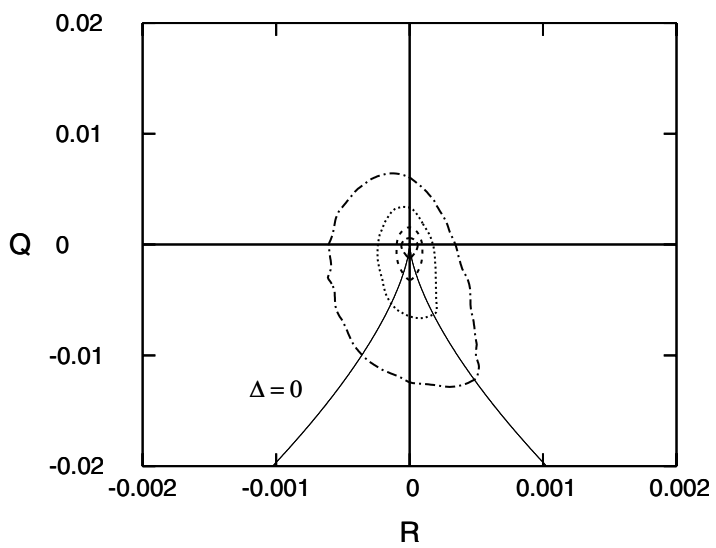

(c)

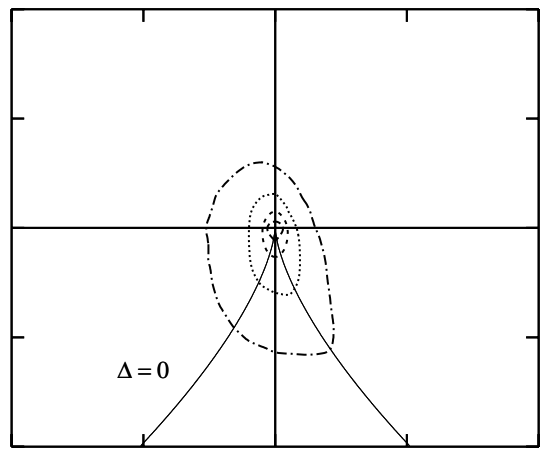

(b)

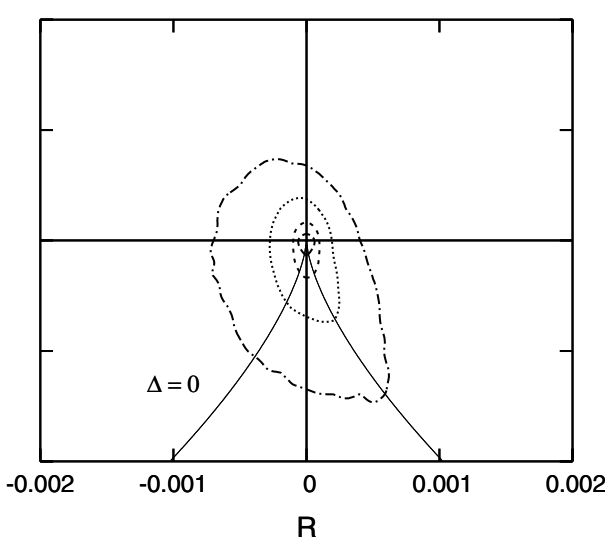

(d)

Fig. 12 Viscous sublayer $\left(z^{+}<5\right)$ joint PDF of Q, R conditionally sampled for fluid $\tau_{p}^{+}=25$ particle positions (b), at $\tau_{p}^{+}=5$ particle positions (c), and at $\tau_{p}^{+}=1$ particle positions (d). Isoline values are: $--\mathrm{PDF}=10,--\mathrm{PDF}=1, \cdots$ $\mathrm{PDF}=0.1,-\cdot-\mathrm{PDF}=0.01$.

To this aim, we can observe that, for incompressible flow, the fluctuating part, $u_{i, j}^{\prime}$, of the velocity gradient tensor computed at the wall has the following form:

$$
\left.u_{i, j}^{\prime}\right|_{w}=\left(\begin{array}{ccc}
0 & 0 & \partial u^{\prime} /\left.\partial z\right|_{w} \\
0 & 0 & \partial v^{\prime} /\left.\partial z\right|_{w} \\
0 & 0 & 0
\end{array}\right)
$$

and the following identities hold: $\left.\tau_{y z}^{\prime}\right|_{w}=\mu \cdot \partial v^{\prime} /\left.\partial z\right|_{w}$ and $\left.\tau_{x z}^{\prime}\right|_{w}=\mu \cdot \partial u^{\prime} /\left.\partial z\right|_{w}$. We already pointed out that the low speed streaks are ejection-like environments that correlate with lower-than-mean wall shear stress regions, where $\left.\tau_{x z}^{\prime}\right|_{w}<0$, and appear much wider than high speed-streaks, associated with higher-than-mean wall shear stress regions, where $\left.\tau_{x z}^{\prime}\right|_{w}>0$. We exploited the relationship between the wall shear stress and the elements of the velocity gradient tensor computed at the wall to describe the near-wall flow regions where particle build-up occurs.

In Fig. 13, we show the instantaneous joint correlations of non-vanishing components of $\left.u_{i, j}^{\prime}\right|_{w}$. We considered the correlations for the fluid (sampled at grid points) and for $\tau_{p}^{+}=1,5$, and 25 particles (sampled at particle positions). Correlations were computed as follows: (i) we considered only the position of particles located in the viscous sublayer - i.e. at $z^{+}<5$; (ii) we projected each particle position onto the wall; (iii) we computed the components of $u_{i, j}^{\prime}$ at the projected wall location. We can observe two distinct near-wall flow regions: With respect to the wall-normal direction, we identify a sweep-like inflow region, characterized by $\left.\tau_{y z}^{\prime}\right|_{w}=0$ associated to $\left.\tau_{x z}^{\prime}\right|_{w}>0$, and an ejection-like outflow region, characterized by $\left.\tau_{y z}^{\prime}\right|_{w}=0$ associated to $\left.\tau_{x z}^{\prime}\right|_{w}<0$. Correlations shown in Figs. 13(b), 13(c), and 13(d) demonstrate that, regardless of particle size, particles in the viscous sublayer tend to accumulate in proximity of wall regions characterized by negative values of $\left.\tau_{x z}^{\prime}\right|_{w}$, i.e. by negative values of $\partial u^{\prime} /\left.\partial z\right|_{w}: 57.5 \%$ of the $\tau_{p}^{+}=1$ particles, $58.4 \%$ of the $\tau_{p}^{+}=5$ particles, and $58.2 \%$ of the $\tau_{p}^{+}=25$ particles fall in the $\partial u^{\prime} /\left.\partial z\right|_{w}<0$ quadrants. 


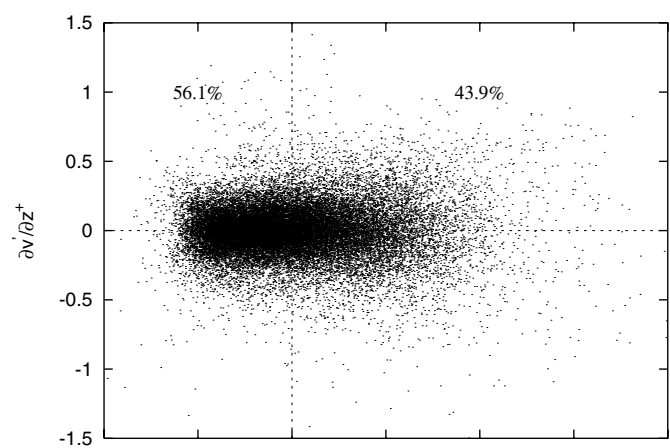

(a)

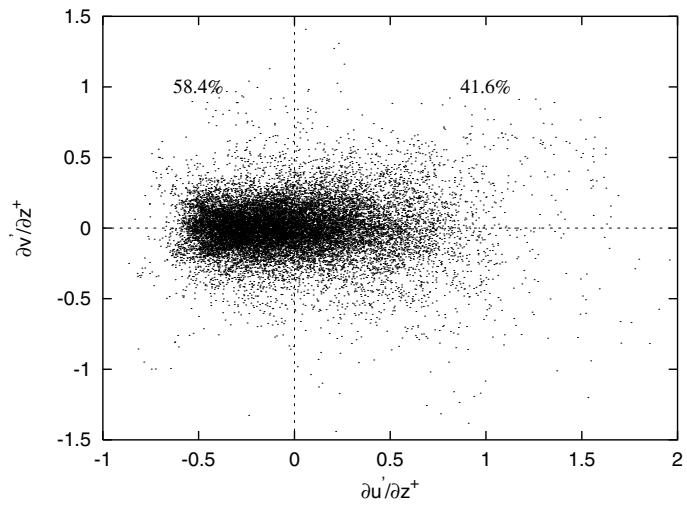

(c)

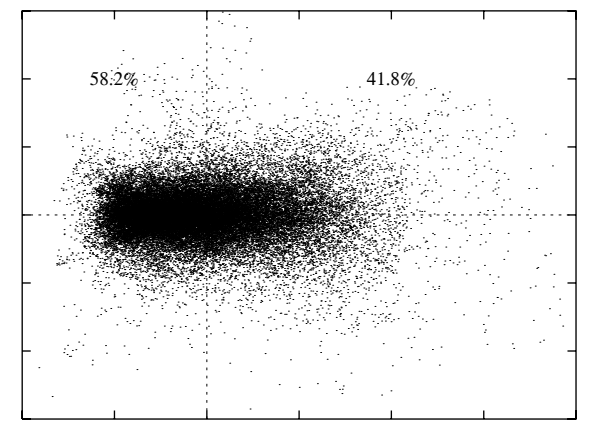

(b)

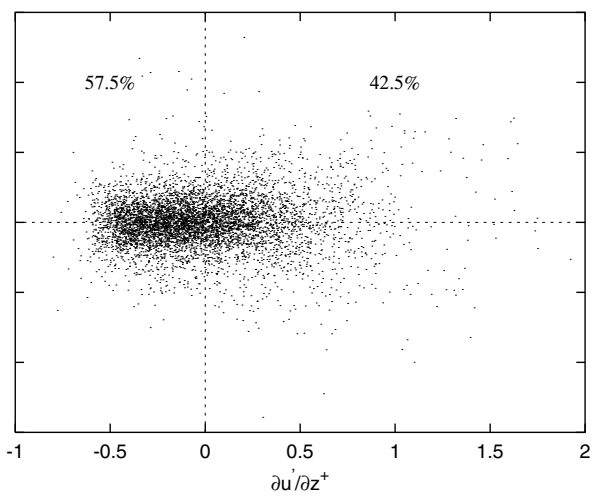

(d)

Fig. 13 Viscous sublayer $\left(z^{+}<5\right)$ instantaneous joint correlations of non-vanishing components of the fluctuating velocity gradient tensor, conditionally sampled at grid points for the fluid (a), and at particle positions projected onto the wall for $\tau_{p}^{+}=25$ particles (b), $\tau_{p}^{+}=5$ particles (c), and $\tau_{p}^{+}=1$ particles (d). Joint correlations demonstrate that particles are mostly concentrated in the ejection-like environments. Percent figures indicate the number of particles in a region of positive/negative $\partial u^{\prime} /\left.\partial z\right|_{w}$.

Even the fluid (see Fig. 13(a)) shows a preferential distribution in such quadrants. This behavior can be explained by considering the mechanisms by which the low-speed streaks are generated: A jet of fluid which is directed to the wall generates the sweep and also the high-speed region; then the jet of fluid, by continuity, is deflected by the wall and generates the low-speed ejection. Due to the entrainment of surrounding fluid, the sweep is more intense and concentrated and the following ejection spreads over a wider cross-section and has lower momentum. Low-speed, low-shear regions, where $\tau_{w}^{\prime}<0$ (i.e. $\partial u^{\prime} /\left.\partial z^{+}\right|_{w}<0$ ), appear much wider than high-speed high-shear regions, where $\tau_{w}^{\prime}>0$ (i.e. $\partial u^{\prime} /\left.\partial z^{+}\right|_{w}>0$ ). Thus, grid points necessarily sample $\partial u^{\prime} /\left.\partial z^{+}\right|_{w}<0$ regions more often than $\partial u^{\prime} /\left.\partial z^{+}\right|_{w}>0$ regions. However, in the latter regions, sampling at grid points appears more scattered and covers a wider range of values of $\partial u^{\prime} /\left.\partial z^{+}\right|_{w}$. The correlations shown in Fig. 13 are similar for all of the 200 realizations of the flow field we studied. This result, together with the fact that the preferred wall regions are also characterized by values of $\partial v^{\prime} /\left.\partial z\right|_{w}$ nearly equal to zero, indicates that particle concentration build-up in the viscous sublayer occurs preferentially in the proximity of a near-wall outflow region. This is examined in detail in a recent work [48] and can be explained considering the crucial role of the quasi-streamwise vortices in trapping particles very near the wall [35].

To provide a unifying pictorial view of the mechanisms discussed by statistical means in this paper, in Fig. 14 the instantaneous snapshot of particle distribution and turbulent coherent structures in the near-wall region is shown, superimposed to the $2 \mathrm{D}$ footprint of the wall shear-stress. The perspective view and the inset top view focus on a window of the computational domain (dimensions are $180 \times 135 \times 55$ in wall units) and give a clear rendering of the physical phenomenon.

Using the $\Omega=0.03$ isosurface with both signs, we have identified two quasi-streamwise vortices: For clarity of presentation, we only show the rear-end of the mature counter-clockwise rotating vortex (blue isosurface) and the forwardend of the secondary clockwise-rotating vortex (red isosurface). As expected, the two vortices are separated by the stremline sheet $\Psi=0$ (green isosurface), cut at $z^{+}=20$. The environment neighboring the streamsurface is an ejection-like outflow region, characterized by low values of the wall shear-stress (in blue). As reported by Marchioli and Soldati [35], this region is squeezed by the two vortices, which act to reduce the probability for particles to be resuspended into the outer flow. 


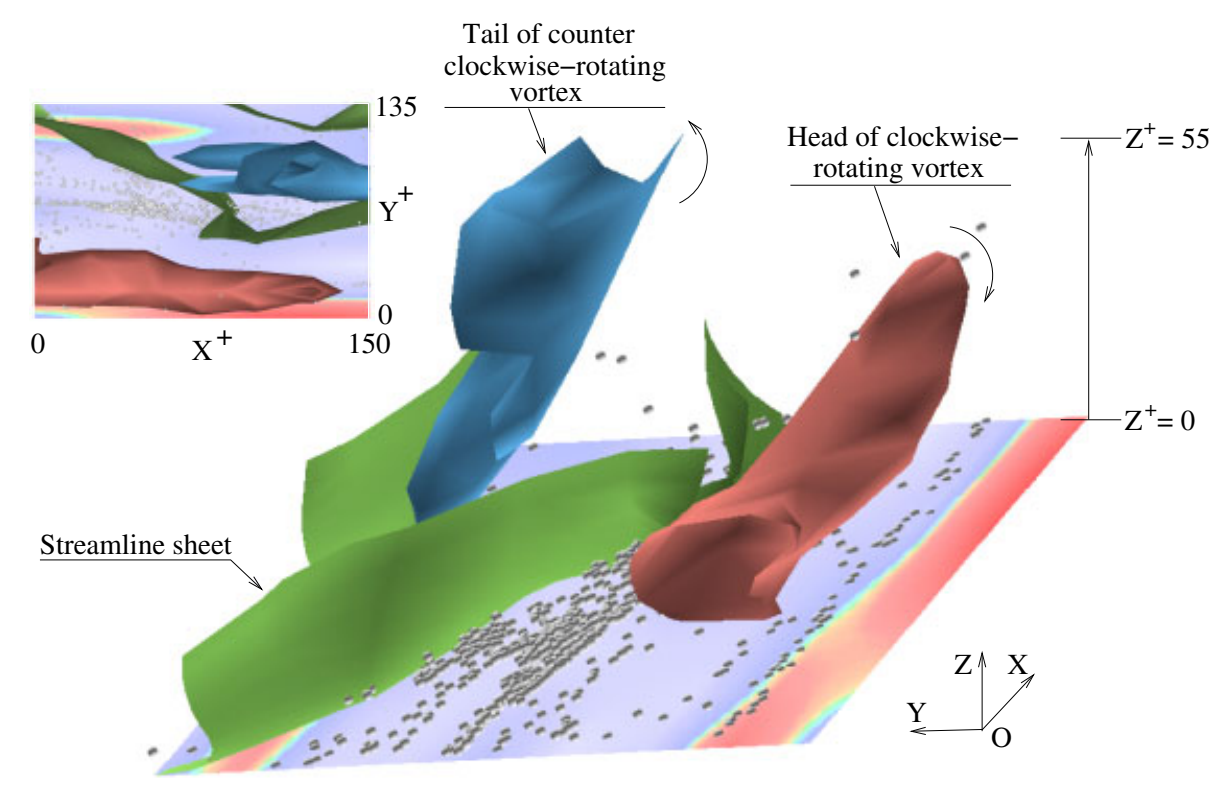

Fig. 14 Instantaneous snapshot of particle distribution and turbulent coherent structures in the near-wall region. Blue isosurfaces indicate counter-clockwise rotating vortices, red isosurfaces indicate clockwise-rotating vortices. Vortices are separated by the $\Psi=0$ streamsurface, in green. Particles, represented as blue spheres, and structures are superposed to the footprint of the wall shear stress: Red indicates high shear-stress, blue indicates low shear-stress.

Most of the particles (plotted as light grey spheres) accumulate in the ejection-like region, avoiding the high shear-stress sweep-like environment at the wall (in red). It is also apparent that coherent vortices are not able to entrain particles. Events shown in Fig. 14 have been observed in a large number of instants at different locations of the channel.

\section{Summary and conclusions}

Particle distribution and transfer mechanisms in turbulent boundary layer is of fundamental significance in a number of engineering and environmental applications. The traditional approach is based on statistical analyses in which particle transfer is modelled with a diffusional - Fickian - process. However, in all cases in which the instantaneous and local particle concentration is the crucial parameter, the time-averaged diffusional approach hides the true physics of the phenomena [59, $60]$.

In this work, we gave a unified view of the transfer mechanisms for particles in turbulent boundary layer. We exploited Direct Numerical Simulation of turbulence and Lagrangian particle tracking to have a fully representative three-dimensional and time-dependent description of the phenomena. We tried to model the cause-effect relationship which control turbulence regeneration cycle in the wall region considering carefully the role of each coherent structure and of each space and time scale involved. We further considered the dispersion of particles characterized by different values of the inertia parameter. Particle inertia is the low-pass filter which influences particle response to turbulence fluctuations - or turbulence coherent structures.

Particle transfer in the wall region of turbulent boundary layers is dominated by the coherent structures which bring them toward the wall and away from the wall and favour particle segregation in the viscous region. Particle fluxes toward and away from the wall strongly depend on the sweep and ejection events. Yet, due to inertia, particle re-entrainment is less effective than particle deposition. We confirmed previous results on particle re-entrainment mechanisms and proved the efficiency of the strongly coherent sweep and ejection events as particle transfer mechanisms. However, we found evidence that strongly coherent ejections are extremely effective in transferring only those particles which are not prevented to reach the region where ejections can entrain them. It is the sincronicity between the strongly coherent ejections and the presence of the rear-end of a quasi-streamwise vortex very near to the wall, which prevents equal in and out wall fluxes which gives reason for the turbophoretic drift phenomenon observed in previous works [67].

We are currently trying [48] to establish a methodology to measure particle preferential distribution in the wall region specifically in the viscous sublayer - exploiting the concept of coherent structure with the classification proposed by Chong et al. [10] and already used by Rouson and Eaton [52] for dispersed flows. We simplified this methodology for specific 
application in the wall region showing preferential concentration in ejection-like outflow regions - characterized by negative values of the streamwise component of the wall shear stress. We considered a simplified version of the phenomenon with particle dynamics influenced by inertia and drag force only and no particle feedback on the flow. This simulation is simplified and yet fully representative of the main qualitative features of the phenomena investigated. For the range of parameters examined - particle dimension, density, and concentration - the effect of other forces as lift, gravity etc. will just add quantitative corrections [35,36]. In dilute flow assumptions, the two-way coupling - particles feedback onto the flow field - will also add just quantitative corrections and the weak flow modulation [26,43] will not modify substantially the quality of the physical model [47]. However, for industrial applications, current research lines will have to consider specifically the influence of particle-particle interactions and of accurate models for particle coalescence and collisions [19,61,63, 64,69].

Acknowledgements I am very much indebted to my coworkers Cristian Marchioli, Andrea Giusti, Maurizio Picciotto, and Marina Campolo. Financial support from MIUR in the frame of PRIN Program under Grant 2003099224_002 and in the frame of FIRB Program under Grant RBAU012FRS_001 and under Grant RBAU01NZH7_007 is gratefully acknowledged.

\section{References}

[1] R. J. Adrian, C. D. Meinhart, and C. D. Tomkins, J. Fluid Mech. 422, 1-54 (2000).

[2] P. S. Bernard, J. M. Thomas, and R.A. Handler, J. Fluid Mech. 253, 385-419 (1993).

[3] H. M. Blackburn, N. N. Mansour, and B. J. Cantwell, J. Fluid Mech. 310, 269-292 (1996).

[4] J.P. Bonnet, J.Delville, M.N. Glauser, R.A. Antonia, D. K. Bisset, D. R. Cole, H.E. Fiedler, J.H. Garem, D. Hilberg, J. Jeong, N. K. R. Kevlahan, L. S. Ukeiley, and E. Vicendeau, Exp. Fluids 25, 197-225 (1998).

[5] J.W. Brooke, K. Kontomaris, T. J. Hanratty, and J. B. McLaughlin, Phys. Fluids A 4, 825-834 (1992).

[6] J.W. Brooke and T. J. Hanratty, Phys. Fluids A 5, 1011-1022 (1993).

[7] M. Campolo, M.V. Salvetti, and A. Soldati, AIChE J. 51, 28-43 (2005).

[8] M. Caporaloni, F. Tampieri, F. Trombetti, and O. Vittori, J. Atmos. Sci. (USA) 32, 565-568 (1975).

[9] S. Cerbelli, A. Giusti, and S. Soldati, Int. J. Multiph. Flow 27, 1861-1879 (2001).

[10] M. S. Chong, A. Perry, and B. J. Cantwell, Phys. Fluids A 2, 765-777 (1990).

[11] J.N. Chung and T. R. Troutt, J. Fluid Mech. 186, 199-222 (1988).

[12] J.W. Cleaver and B. Yates, Chem. Eng. Sci. 30, 983-992 (1975).

[13] C.T. Crowe, M. Sommerfeld, and Y. Tsuji, Multiphase flows with droplets and particles (CRC Press, NY, 1998).

[14] V. De Angelis, P. Lombardi, P. Andreussi, and S. Banerjee Microphysics of scalar transfer at air-water interfaces. Invited Paper, IMA Conference on Wind over Wave Couplings, Salford, UK, 8-10 April, 1997 (Oxford University Press, 1997).

[15] Y. Dubief and F. Delcayre, J. Turbul. 1, 11-32 (2000).

[16] J. K. Eaton and J. R. Fessler, Int. J. Multiph. Flow 20, 169-209 (1994).

[17] S. K. Friedlander and H. F. Johnstone, Ind. Eng. Chem. Res. 49, 1151-1156 (1957).

[18] Y.G. Guezennec and W. C. Choi, in: Proc. of Zoran P. Zaric Memorial International Seminar on Near Wall Turbulence, edited by S. J. Kline and N.H. Afghan (Hemisphere, New York, 1989), pp. 420-436.

[19] C. A. Ho and M. Sommerfeld, Chem. Eng. Sci. 57, 3073-3084 (2002).

[20] J. C. R. Hunt, A.A. Wray, and P. Moin, Center of Turbulence Research Rep. CTR-S88, 193 (1998).

[21] F. Hussain, Phys. Fluids 26, 2816-2838 (1983).

[22] F. Hussain, J. Fluid Mech. 173, 303-356 (1986).

[23] J. Jeong and F. Hussain, J. Fluid Mech. 285, 69-83 (1995).

[24] J. Jeong, F. Hussain, W. Schoppa, and J. Kim, J. Fluid Mech. 332, 185-214 (1997).

[25] J. Jimenez and A. Pinelli, J. Fluid Mech. 389, 335-359 (1999).

[26] D. Kaftori, G. Hetsroni, and S. Banerjee, Phys. Fluids 7, 1107-1121 (1995).

[27] N. Kasagi and O. Iida Progress in Direct Numerical Simulation of Turbulent Heat Transfer. Keynote Paper, 5th ASME/JSME Joint Thermal Engineering Conference, San Diego, CD-ROM Publication, ASME, March, 1999.

[28] J. Kim, P.Moin, and R. Moser, J. Fluid Mech. 177, 133-166 (1987).

[29] J. Kim and F. Hussain, Phys. Fluids A 5, 695-706 (1993).

[30] S. J. Kline, W. C. Reynolds, F. A. Schraub, and P. W. Runstadler, J. Fluid Mech. 70, 741-773,(1967).

[31] S. J. Kline, S. K. Robinson, and P. R. Spalart, in: Proc. of Zoran P. Zaric Memorial International Seminar on Near Wall Turbulence, edited by S. J. Kline and N. H. Afgan (Hemisphere, New York, 1989), pp. 218-247.

[32] K. Lam and S. Banerjee, Phys. Fluids A 4, 306-320 (1992).

[33] E. Loth, Progress Energy Combustion Science 26, 161-223 (2000).

[34] S. L. Lyons, T. J. Hanratty, and J. B. McLaughlin, Int. J. Numer. Meth. Fluids 13, 999-1028 (1991).

[35] C. Marchioli and A. Soldati, J. Fluid Mech. 468, 283-315 (2002).

[36] C. Marchioli, A. Giusti, M. V. Salvetti, and A. Soldati, Int. J. Multiph. Flow 29, 1017-1038 (2003).

[37] M. R. Maxey and J. J. Riley, Phys. Fluids 26, 883-889 (1983).

[38] M. R. Maxey, Phys. Fluids 30, 1915-1928 (1987).

[39] J.B. McLaughlin, Phys. Fluids 1, 1211-1224 (1989).

[40] G. Mungal, Personal communication, Udine (2001). 
[41] C. Narayanan, D. Lakehal, L. Botto, and A. Soldati, Phys. Fluids A 15, 763-775 (2003).

[42] Y. Niño and M.H. Garcia, J. Fluid Mech. 326, 285-319 (1996).

[43] Y. Pan and S. Banerjee, Phys. Fluids 8, 2733-2755 (1996).

[44] D. V. Papavassiliou and T. J. Hanratty, Int. J. Heat Fluid Flow 18, 55-69 (1997).

[45] S. Pedinotti, G. Mariotti, and S. Banerjee, Int. J. Multiph. Flow 18, 927-941 (1992).

[46] A. Perry and M. S. Chong, Ann. Rev. Fluid Mech. 9, 125-148 (1987).

[47] M. Picciotto, C. Marchioli, and A. Soldati, Coupling mechanisms between turbulence and microparticles in turbulent boundary layer, 16th Int. Conf. on Chemical and Process Engineering, Prague, CZ, 22-26 Aug 2004.

[48] M. Picciotto, C. Marchioli, and A. Soldati, Characterization of near-wall accumulation regions for inertial particles in turbulent boundary layers, submitted (2005).

[49] L. M. Portela, P. Cota, and R.V.A. Oliemans, Powder Technol. 125, 149-157 (2002).

[50] M.W. Reeks, J. Aerosol Sci. 14, 729-739 (1983).

[51] S. K. Robinson, Ann. Rev. Fluid Mech. 23, 601-639 (1991).

[52] D. W. I. Rouson and J. K. Eaton, J. Fluid Mech. 428, 149-169 (2001).

[53] F. Sbrizzai, R. Verzicco, M. Pidria and A. Soldati, Int. J. Multiph. Flow 30, 1389-1417 (2004).

[54] V.L. Schiller and A. Naumann, VDI-Z 77, 318-320 (1933).

[55] W. Schoppa and F. Hussain, in: Eddy Structure Identification, edited by J.P. Bonnet, CISM Courses and Lectures 353 (SpringerVerlag, Wien, 1996), pp. 61-143.

[56] W. Schoppa and F. Hussain, in: Self-sustaining Mechanisms of Wall Turbulence, edited by R. Panton (Computational Mechanics Publications Vol. 15), (WIT Press, Shouthamton, 1997), pp. 385-422.

[57] W. Schoppa and F. Hussain, J. Fluid Dyn. Res. 26, 119-139 (2000).

[58] W. Schoppa and F. Hussain, J. Fluid Mech. 453, 57-108 (2002).

[59] A. Soldati, J. Aerosol Sci. 31, (2000), 293-305.

[60] A. Soldati, Aerosol Sci. Technol. 37, 171-182 (2003).

[61] A. Soldati and P. Andreussi, Chem. Eng. Sci. 51, 353-363 (1996).

[62] A. Soldati, and S. Banerjee, Phys. Fluids 10, 1742-1756 (1998).

[63] M. Sommerfeld, Int. J. Multiph. Flow 29, 675-699 (2003).

[64] M. Sommerfeld and J. Kussin, Int. J. Multiph. Flow 29, 701-718 (2003).

[65] W. S. J. Uijttewaal and R.V.A. Oliemans, Phys. Fluids 8, 2590-2604 (1996).

[66] W.W. Willmarth and S. S. Lu, J. Fluid Mech. 55, 65-92 (1972).

[67] B. Young and T. J. Hanratty, Am. Inst. Chem. Eng. J. 37, 1529-1536 (1991).

[68] J. Young and A. Leeming, J. Fluid Mech. 340, 129-159 (1997).

[69] L. I. Zaichik, O. Simonin, and V. M. Alipchenkof, Phys. Fluids 15, 2995-3005 (2003).

[70] H. Zhang and G. Ahmadi, J. Fluid Mech. 406, 55-80 (2000).

[71] J. Zhou, R. J. Adrian, S. Balachandar, and T. M. Kendall, J. Fluid Mech. 387, 353-396 (1999).

\section{Appendix: Streamline rotation vector}

The recognition of coherent structures in turbulence is a puzzling issue since no widely accepted definition of coherent structure exists. Most definitions involve subjective choices of cut-off values or reference frames. For example, local pressure minima below some threshold value are often used to identify vortex cores. One scheme for rational selection of the cut-off values was developed by Chong, Perry, and Cantwell [10]. Their scheme involves the examination of every point in the flow domain from a reference frame translating with the local fluid velocity. Each point is then a critical point of the dynamical system obtained by Taylor expansion of the instantaneous fluid velocity $\mathbf{u}$ about a point $\mathbf{x}$. For all case of practical interest, the phase-space trajectory of such dynamical system, and hence the physical-space trajectory of fluid elements, is governed by a three-dimensional set of first-order differential equations, written as:

$$
\dot{x}=A x,
$$

where:

$$
\dot{x}=\left(\begin{array}{c}
\dot{x}_{1} \\
\dot{x}_{2} \\
\dot{x}_{3}
\end{array}\right), A=\left(\begin{array}{ccc}
a_{11} & a_{12} & a_{13} \\
a_{21} & a_{22} & a_{23} \\
a_{31} & a_{32} & a_{33}
\end{array}\right), x=\left(\begin{array}{c}
x_{1} \\
x_{2} \\
x_{3}
\end{array}\right)
$$

Elements $a_{i j}$ of matrix $A$ are real constants. In the case of a fluid flow, the $a_{i j}$ are the elements of the rate-of-deformation tensor $\partial \dot{x}_{i} / \partial x_{j}=\partial u_{i} / \partial x_{j}=u_{i, j}$, where $u_{i}$ and $x_{i}$ are the $i$ th Cartesian components of the vectors $\mathbf{u}$ and $\mathbf{x}$, the subscript comma symbolizes partial differentiation with respect to the subsequent index, and Einstein summation convention applies.

Let us now split the rate-of-deformation tensor into a symmetric $S_{i j}$ and antisymmetric part $\Omega_{i j}$ defined as follows:

$$
S_{i j}=\frac{1}{2}\left(u_{i, j}+u_{j, i}\right), \Omega_{i j}=\frac{1}{2}\left(u_{i, j}-u_{j, i}\right) .
$$




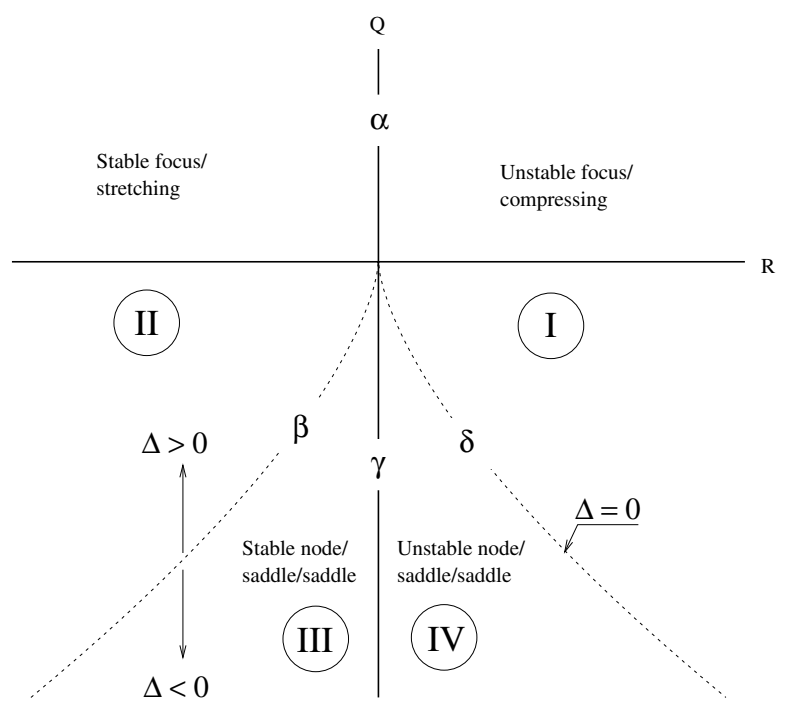

Fig. 15 Incompressible flow critical point topologies according to the classification scheme of Chong et al. [10].

$S_{i j}$ is the rate-of-strain tensor and describes the rate at which an infinitesimal control volume of fluid changes shape. $\Omega_{i j}$ is the rotation (or spin) tensor: $S_{i j}$ and $\Omega_{i j}$ represent the local balance between shear strain rate and vorticity magnitude. Shear strain and vorticity have the same magnitude at a stationary wall and thus the rate-of-deformation tensor vanishes at the wall. If $\lambda_{1}, \lambda_{2}, \lambda_{3}$ are the eigenvalues of matrix $A$, then:

$$
[A-\lambda I] \mathbf{e}=0
$$

where $\mathbf{e}$ is the eigenvector. The eigenvalues can be determined by solving the characteristic equation $\operatorname{det}[A-\lambda I]=0$ which, for a $3 \times 3$ matrix, can be written as $\lambda^{3}-P \lambda^{2}+Q \lambda-R=0$, where:

$$
\begin{aligned}
& P=a_{11}+a_{22}+a_{33}=-\operatorname{tr}[A]=-S_{i i}, \\
& Q=\left|\begin{array}{ll}
a_{11} & a_{12} \\
a_{21} & a_{22}
\end{array}\right|+\left|\begin{array}{ll}
a_{11} & a_{13} \\
a_{31} & a_{33}
\end{array}\right|+\left|\begin{array}{ll}
a_{22} & a_{23} \\
a_{32} & a_{33}
\end{array}\right| \\
& =\frac{1}{2}\left(P^{2}-\operatorname{tr}\left[A^{2}\right]\right)=\frac{1}{2}\left(P^{2}+\Omega_{i j} \Omega_{i j}-S_{i j} S_{i j}\right),
\end{aligned}
$$

and

$$
\begin{aligned}
& R=\left|\begin{array}{lll}
a_{11} & a_{12} & a_{13} \\
a_{21} & a_{22} & a_{23} \\
a_{31} & a_{32} & a_{33}
\end{array}\right|=\operatorname{det}[A]=\frac{1}{3}\left(P^{3}-3 P Q+\operatorname{tr}\left[A^{3}\right]\right) \\
& =\frac{1}{3}\left(P^{3}-3 P Q+S_{i j} S_{j k} S_{k i}+3 \Omega_{i j} \Omega_{j k} \Omega_{k i}\right),
\end{aligned}
$$

are the first, second, and third invariants of $A$. Recall that the characteristic equation can have (i) all real roots which are distinct, (ii) all real roots where at least two roots are equal, or (iii) one real root and a conjugate pair of complex roots.

Chong et al. [10] classified all possible local streamline patterns around any point in a flow (in a reference frame moving with the velocity of that point) according to the values of the three invariants of the rate-of-deformation tensor $(P, Q$, and $R$ ), i.e. according to the values of eigenvalues $\lambda_{i}$. They proposed that a vortex core is a region with complex eigenvalues of $\partial u_{i} / \partial x_{j}$ : From a physical viewpoint, complex eigenvalues imply a local swirling motion so that the local streamline pattern is closed or spiral in a reference frame moving with the point. In other words, a vortex core is a region where the vorticity is sufficiently strong to cause the rate-of-deformation tensor to be dominated by the rotation tensor.

For incompressible flow, $P=0$ due to mass conservation, $Q=-\frac{1}{2} u_{i, j} u_{j, i}$ and $R=\operatorname{det}\left(u_{i, j}\right)$. Thus complex eigenvalues will occur when the discriminant $\Delta$ of the characteristic equation is positive:

$$
\Delta=\left(\frac{1}{3} Q\right)^{3}+\left(\frac{1}{2} R\right)^{2}>0 .
$$


For visualization purposes, the vector $\Omega$ is computed only at those point where $\Delta>0$ by means of the following equation:

$$
\boldsymbol{\Omega}=\operatorname{Im}\left(\lambda_{c}\right) \frac{\mathbf{e}_{\lambda_{r}}}{\left|\mathbf{e}_{\lambda_{r}}\right|} \frac{\mathbf{e}_{\lambda_{r}} \cdot\left[\operatorname{Re}\left(\mathbf{e}_{\lambda_{c}}\right) \times \operatorname{Im}\left(\mathbf{e}_{\lambda_{c}}\right)\right]}{\left|\mathbf{e}_{\lambda_{r}} \cdot\left[\operatorname{Re}\left(\mathbf{e}_{\lambda_{c}}\right) \times \operatorname{Im}\left(\mathbf{e}_{\lambda_{c}}\right)\right]\right|},
$$

where $\operatorname{Im}\left(\lambda_{c}\right)$ is the imaginary part of the pair of complex eigenvalues, $\operatorname{Re}\left(\mathbf{e}_{\lambda_{c}}\right)$ and $\operatorname{Im}\left(\mathbf{e}_{\lambda_{c}}\right)$ are the real part and the imaginary part of the conjugate complex eigenvectors corresponding to the complex eigenvalues, $\mathbf{e}_{\lambda_{r}}$ is the eigenvector corresponding to the real eigenvalue $\lambda_{r}$.

The rate-of-deformation invariants can be used to provide a classification of elementary three-dimensional flow patterns defined by instantaneous streamlines for flow at and away from no-slip boundaries [3,10]. This scheme provides an objective classification that eliminates the arbitrary choice of threshold values used in other schemes.

For incompressible flow, all possible topologies can be classified in the $(Q, R)$-plane, as shown in Fig. 15. Considering the $\Delta=0$ curve, the $(Q, R)$-plane is divided into four regions: the so-called stable focus/stretching, unstable focus/compressing, stable node/saddle/saddle and unstable node/saddle/saddle critical points. The first two are vortical flow regions, the last two are essentially convergence zones. 